\title{
Variational Principle for Relativistic Fluid Dynamics
}

\author{
Hans-Thomas Elze ${ }^{1}$, Yogiro Hama ${ }^{2}$, Takeshi Kodama ${ }^{1}$, Martín Makler $^{3}$ and Johann Rafelski ${ }^{4}$ \\ ${ }^{1}$ Instituto de Física, Universidade Federal do Rio de Janeiro, \\ CP 68.528, 21945-970 Rio de Janeiro, Brasil \\ ${ }^{2}$ Instituto de Física, Universidade de São Paulo, CP 66318, \\ 05389-970 Sao Paulo, Brasil \\ ${ }^{3}$ Centro Brasileiro de Pesquisas Físicas, Rua Xavier Sigaud 150, \\ 22290-160 Rio de Janeiro Brasil \\ ${ }^{4}$ Physics Department, University of Arizona, Tucson, AZ 85721 USA
}

\begin{abstract}
The variational principle for the special and general relativistic hydrodynamics are discussed in view of its application to obtain approximate solutions to these problems. We show that effective Lagrangians can be obtained for suitable ansatz for the dynamical variables such as density profile of the system. As an example, the relativistic version of spherical droplet motion (Rayleigh-Plesset equation) is derived from a simple Lagrangian. For the general relativistic case the most general Lagrangian for spherically symmetric systems is given.
\end{abstract}

\section{INTRODUCTION}

First applications of relativistic hydrodynamics to the process of multiparticle production in high-energy hadronic collisions can be found in the works of Fermi and Landau in the early 1950's [1.22. Recently, extensive studies of the relativistic motion of fluids have been done with respect to the analysis of relativistic heavy-ion collision processes [3 5]. In fact, a hydrodynamic description of high-energy hadronic and nuclear collisions has been successful in reproducing global features of these processes, such as multiplicity and transverse energy distributions. From the theoretical point of view, however, the foundation of the hydrodynamical picture for these processes is not a trivial matter. This is because the use of hydrodynamic equations of motion assumes implicitly the local thermal equilibrium via an equation of state of the matter. This means that the relaxation time scale and the mean free path should be much smaller compared to, respectively, the hydrodynamical time scale and spatial size of the system. In this sense, one may wonder whether these conditions could easily be met for hadronic and nuclear collisions (for the collision of heavier nuclei they are expected to be approximately fulfilled for some specific scenario).

On the other hand, from the kinematical point of view, apart from the use of the equation of state, the equations of hydrodynamics are nothing but the conservation laws of energy and momentum, together with other conserved quantities such as charge. In this sense, for any process where the dynamics of flow is an important factor, a hydrodynamic framework should be a natural first step, at least at the level of phenomenology. The effects of finite relaxation time and mean-free path might be implemented at a later stage by using an effective equation of state, incorporating viscosity and heat conductivity, or some simplified transport equations, see Ref. [6] and references therein.

Another important arena of extensive application of relativistic hydrodynamics is found in cosmology and highenergy astrophysics, such as the gravitational collapse of a stellar core to form a neutron star or a black hole, relativistic blast waves for the models of gamma ray bursts, etc. [讶 10]. In these cases, the assumption of the local thermodynamical equilibrium is considered to be well justified. However, in the astrophysical applications we have not only to face the large scale systems but also to deal with the long range gravitational field simultaneously. For these reasons, the computer simulations of hydrodynamical scenarios for astrophysical problems usually become extremely expensive.

The relativistic hydrodynamics is a local description of the conservation laws, written in terms of the energymomentum tensor as

$$
\partial_{\mu} T^{\mu \nu}=0 .
$$

This is a set of coupled partial differential equations which, in general, are difficult to solve exactly. Except for a few analytical solutions known for special cases, we have usually to resort to numerical solutions even for a simplest geometry, like one-dimensional or spherically symmetric cases. In the most of the cases, the numerical approach together with a realistic equation of state becomes prohibitively expensive, especially when coupled to some transport equations such as those for neutrinos in the case of stellar collapse or supernova explosions [11.

In addition to the difficulties of solving the hydrodynamical equations, frequently we encounter with the situation where even the equation of state of the matter is not known precisely. Rather, we apply the hydrodynamical models to infer the properties of the matter involved in the process. In such cases we do not need the very precise local 
features of the hydrodynamical motion (for example, sound ripples, small local perturbations, etc.) but rather the global flow motion which characterizes the dynamics of the system assuming a given equation of state.

For the reasons cited above, in spite of the presence of highly sophisticated techniques for hydrodynamic numerical calculations, some problems require rather simpler approaches which allow to analyze the dynamics of the system more effectively. In such cases, extremely local properties should be smeared out effectively, in order to extract global features of the flow more directly. As an example, we know that some global features of the high-energy hadronic and nuclear collisions can already be described by a simple fireball model [12]. Presently we aim at a dynamical scheme which improves the simplest fireball model in the direction of a more complete hydrodynamical description.

We here introduce the method of effective action based on the variational principle to hydrodynamic equations of motion. As is well-known, the variational approach has practical advantages besides its formal side. Once the variational principle is established, we can use the method to obtain the optimal parameters of a given family of trial solutions. The effective Lagrangian and variational approach [13,14, introduced to incorporate the effect of local turbulent motion in an effective way into a supernova explosion mechanism, is such an example. It was shown that such an approach is also useful to discuss the dynamics of a sonoluminescencing bubble in a fluid 15, 16. There, the effective Lagrangian method was shown to be very useful in generalizing the so-called Rayleigh-Plesset equation to include in a simple way the effects of gas dynamics inside the bubble. There exist some analogous problems to the dynamics of a sonoluminescence bubble in the domain of relativistic energies, such as QGP or astrophysical fireballs. Thus, the relativistic generalization of the classical Rayleigh-Plesset equation will be useful.

In the present work, we generalize the effective Lagrangian method to the relativistic hydrodynamics. By suitable parametrizations of the density profile of the system, approximate but very simple solutions of relativistic hydrodynamical models can be derived in this approach. In particular, we derive a relativistic generalization of the Rayleigh-Plesset equation and discuss the effect of relativity for the homologous motion of gas and fluid.

Frequently the flow of the matter accompanies the production of entropy. In particular, when a shock wave is generated, the violent dynamical change of the density leads to a highly turbulent regime, which cascades into a smaller scale complex fluid motion and ultimately thermalize. In order to incorporate such effects of non-adiabatic processes and simulate the dynamics of shock wave as a thin domain of non-adiabatic flow, Neumann and Richtmyer 117 introduced the method of the pseudo-viscosity which is still used extensively in many areas. We show that this approach can well be incorporated in our formalism and consequently, the relativistic generalization of the pseudoviscosity method is easily obtained in our context.

In the astrophysical applications of relativistic hydrodynamics, the inclusion of the gravitational field is essential. It has been discussed by several authors that the general relativistic hydrodynamical equations can also be derived from the action principle 18 21. In this paper, we derive a simple general relativistic effective Lagrangian for spherically symmetric systems and deduce explicitly from it the equation of motion of Misner and Sharp [22] for gravitationally collapsing object. We also show that the concept of general coordinate system allows us to use a comoving Lagrangian frame in obtaining the effective Lagrangian of the special relativistic hydrodynamics.

We organize this paper as follows. In Sec. II, we first review the variational formulation of the relativistic hydrodynamics. Then, in Sec.III, we apply the variational scheme to spherically symmetric cases and establish an effective Lagrangian for the variational parameters of the density profile function. In the case of a homogeneous gas bubble in an infinite fluid, this equation is a relativistic generalization of the Rayleigh-Plesset equation well-known for fluid acoustic theories. In Sec. IV, we discuss nonadiabatic process and generalize the pseudo-viscosity of Neumann and Richtmeyer in the context of our relativistic variational principle. In Sec.V, we extend our approach to the general relativistic case, where, the metric functions are chosen as the dynamical variables. In the case of spherically symmetric system, we derive explicitly the effective Lagrangian for the comoving coordinate system. We discuss the relation between the comoving frame and space-fixed coordinate system. In Sec.VI, we summarize our present work.

\section{VARIATIONAL APPROACH}

Although not commonly found in general textbooks, the variational formulation of hydrodynamics has been studied by several authors 18 21,23 26]. For the sake of later discussion, let us first review briefly how the relativistic hydrodynamical equations of motion are derived from a variational principle. In the following, we take the velocity of light is unity, $c=1$. Let the velocity field of the matter be

$$
\vec{v}=\vec{v}(\vec{r}, t) .
$$

In order to keep the manifestly covariant notation, we express the flow in terms of a four-vector, $u^{\mu}(x)$, where

$$
u^{0}=\gamma, \quad \vec{u}=\gamma \vec{v} .
$$


and

$$
u_{\mu} u^{\mu}=1
$$

The flow of matter induces a change in the specific volume occupied by the matter. In order to facilitate the following discussion, we consider the case where there exists some conserved quantity, say the baryon number. Let the local density of this conserved quantity in the comoving frame be $n$. Then we have

$$
\partial_{\mu}\left(n u^{\mu}\right)=0 .
$$

We also define the specific volume $V$ as

$$
V=\frac{1}{n}
$$

Let us write the energy of the matter in this volume as

$$
E=\varepsilon V,
$$

where $\varepsilon$ is the energy density. The assumption of local equilibrium leads to the validity of the thermodynamical relations, such as

$$
\left(\frac{\partial E}{\partial V}\right)_{S}=-P
$$

where $S$ is the entropy of the matter in the volume and $P$ the pressure. In terms of the energy density this implies

$$
\left(\frac{\partial \varepsilon}{\partial n}\right)_{S}=\frac{\varepsilon+P}{n} .
$$

Now take the action,

$$
I_{M}=\int d^{4} x\left\{-\varepsilon(n)+\xi(x) \partial_{\mu}\left(n u^{\mu}\right)+\frac{1}{2} \zeta(x)\left(u^{\mu} u_{\mu}-1\right)\right\} .
$$

and state the variational principle as

$$
\delta I_{M}=0
$$

for arbitrary variations in $u^{\mu}, n, \xi$, and $\zeta$. Then, as we will show in the following, Euler's equation for the relativistic fluid motion can be derived formally from the variational principle円. Note that the last two terms in Eq. (10) represent the constraints among variables $u^{\mu}$ and $n$. As we see in the next section, for the practical usage of this variational approach, it is convenient to choose the parametrization of $u^{\mu}$ and $n$ in such a way that the constraints are automatically satisfied so that the Lagrangian multipliers $\xi$ and $\zeta$ do not enter into the calculation.

The variations in $\xi$ and $\zeta$ lead immediately to the constraints, Eqs.(4) and (5). Applying an integration by parts to the second term in Eq.(10), the action can also be written as

$$
I_{M}=\int d^{4} x\left\{-\varepsilon(n)-n u^{\mu} \partial_{\mu} \xi(x)+\frac{1}{2} \zeta(x)\left(u^{\mu} u_{\mu}-1\right)\right\}
$$

We remark that the derivation in this section is equally valid in general coordinate systems. In this case, the partial derivative, $\partial^{\mu}$, in Eq.(11) should be replaced by the appropriate covariant derivative, and correspondingly in the following equations. Furthermore, the volume element $d^{4} x$ should be replaced by the invariant volume element $\sqrt{-g} d^{4} x[27]$.

The variation with respect to $n$ leads to

\footnotetext{
${ }^{1}$ In fact, the action above only applies to the case of non-rotational flow. It is also possible to formulate the variational principle for more general flow pattern. See [19,20] for discussion.
} 


$$
-\frac{\delta \varepsilon}{\delta n}-u^{\mu} \partial_{\mu} \xi=0
$$

Note that, if the motion of the fluid is not adiabatic, then $\delta \varepsilon / \delta n$ does not necessarily be equal to the usual derivative $d \varepsilon / d n$ (see the later discussion). On the other hand, the variation in $u^{\mu}$ leads to

$$
\partial_{\mu} \xi=\frac{1}{n} \zeta u_{\mu},
$$

and substituting this into Eq.(12), we obtain:

$$
\zeta=-n \frac{\delta \varepsilon}{\delta n}
$$

where we have used $u^{2}=1$, cf. Eq.(4). Thus, Eq.(13) is rewritten as

$$
\partial_{\mu} \xi=-\frac{\delta \varepsilon}{\delta n} u_{\mu} .
$$

Taking the contraction of both sides with the four-velocity, we have

$$
u^{\mu} \partial_{\mu} \xi=-\frac{\delta \varepsilon}{\delta n} u_{\mu} u^{\mu}=-\frac{\delta \varepsilon}{\delta n} .
$$

Thus we conclude that

$$
\frac{\partial \xi}{\partial \tau}=-\frac{\delta \varepsilon}{\delta n}
$$

since $u^{\mu} \partial_{\mu} \xi=\partial \xi / \partial \tau$.

In the usual hydrodynamic equations, we assume that the matter is always in thermodynamical equilibrium. Furthermore, if there is no viscosity or heat conduction, the energy change associated to the motion is adiabatic, that is, the change in specific energy $E$ caused by the change in the specific volume $V$ is given by

$$
\delta E=-P \delta V,
$$

where $V=1 / n$ and $P$ is the pressure. In such cases, we have

$$
\frac{\partial \varepsilon}{\partial n} \rightarrow \frac{\delta(n E)}{\delta n}=\frac{\varepsilon+P}{n}
$$

and

$$
d\left(\frac{\delta \varepsilon}{\delta n}\right) \rightarrow \frac{1}{n} d P
$$

Therefore, for adiabatic changes of the density $n$, Eq.(17) becomes

$$
\partial_{\mu} \frac{\partial \xi}{\partial \tau}=-\frac{1}{n} \partial_{\mu} P .
$$

On the other hand, we have also

$$
\begin{aligned}
\partial_{\mu} \frac{\partial \xi}{\partial \tau} & =\partial_{\mu}\left(u^{\nu} \partial_{\nu} \xi\right)=u^{\nu} \partial_{\mu} \partial_{\nu} \xi+\left(\partial_{\mu} u^{\nu}\right) \partial_{\nu} \xi \\
& =u^{\nu} \partial_{\nu} \partial_{\mu} \xi-\frac{\varepsilon+P}{n} u_{\nu}\left(\partial_{\mu} u^{\nu}\right) \\
& =\frac{\partial}{\partial \tau} \partial_{\mu} \xi-\frac{\varepsilon+P}{n} \partial_{\mu}\left(\frac{1}{2} u_{\nu} u^{\nu}\right)=\frac{\partial}{\partial \tau} \partial_{\mu} \xi,
\end{aligned}
$$

that is, the two derivatives $\partial_{\mu}$ and $\partial / \partial \tau$ commute when applied to $\xi$. Therefore, from Eqs.(15) and (17) we can eliminate $\xi$ to get 


$$
u^{\nu} \partial_{\nu}\left[\frac{\varepsilon+P}{n} u_{\mu}\right]=\frac{\partial_{\mu} P}{n} .
$$

Again using Eq.(20), this reduces to

$$
u_{\mu} u^{\nu}\left(\partial_{\nu} P\right)+(\varepsilon+P) u^{\nu} \partial_{\nu} u_{\mu}=\partial_{\mu} P \text {. }
$$

The first term of this equation is further modified as

$$
\begin{aligned}
u^{\nu}\left(\partial_{\nu} P\right) & =u^{\nu} n \partial_{\nu}\left[\frac{\varepsilon+P}{n}\right] \\
& =u^{\nu} \partial_{\nu}(\varepsilon+P)-\frac{\varepsilon+P}{n} u^{\nu} \partial_{\nu} n \\
& =u^{\nu} \partial_{\nu}(\varepsilon+P)+(\varepsilon+P) \partial_{\nu} u^{\nu}
\end{aligned}
$$

where the continuity equation (5) was used. Thus, Eq.24) can be rewritten as

$$
\partial_{\nu} T_{\mu}^{\nu}=0
$$

where

$$
T_{\mu \nu}=(\varepsilon+P) u_{\mu} u_{\nu}-P g_{\mu \nu} .
$$

That is, we arrive at the equation of motion of relativistic fluid dynamics with the energy-momentum tensor of the perfect fluid. From this equation, we obtain the relativistic version of the Euler equation [27],

$$
\frac{\partial}{\partial t} \vec{v}+(\vec{v} \cdot \nabla) \vec{v}=-\frac{1}{(\varepsilon+P) \gamma^{2}}\left[\nabla P+\vec{v} \frac{\partial P}{\partial t}\right] .
$$

In the above derivation, we assumed the Minkowski space-time metric, but as mentioned before all the calculations can easily be extended to the case where the metric is more general. For example, the final result Eq.(26) in the curved metric is

$$
T_{\mu ; \nu}^{\nu}=0
$$

where ; stands for the covariant derivative, as usual.

As pointed out by several authors 19,20,26, the above scheme leads only to non-rotational flow. This can be seen from Eq.(13), where the velocity field is proportional to the four-gradient of a scalar function. In order to include the rotational flow, we have to add a term coming from another constraint with respect to entropy in the original action. However, for the spherically symmetric case below, one does not need to worry about the rotational flow, so we omit the discussion for the sake of simplicity.

\section{SPHERICALLY SYMMETRIC CASE}

The above variational approach is particularly useful when we can solve the constraint equations explicitly. One dimensional, or spherically symmetric system is such a case. Here we study the spherically symmetric case. Let the density profile in a space fixed frame be

$$
\bar{n}=f(r, t) .
$$

Then the velocity field is determined from the continuity equation as

$$
v=-\frac{1}{r^{2} f} \int_{0}^{r} r^{2} \dot{f} d r .
$$

If we use this expression for the velocity field, then we can omit the constraint terms in the action. Thus, we have a Lagrangian for $f$ as

$$
L=-4 \pi \int_{0}^{\infty} r^{2} d r \varepsilon(n)
$$


where the local density in the comoving frame is given by

$$
n=\frac{f}{\gamma}
$$

To see explicitly how the variational principle works with this Lagrangian, let us consider an arbitrary density variation,

$$
f \rightarrow f+\delta f
$$

Under such variation, we get

$$
\begin{aligned}
\delta I & =-4 \pi \int d t \int_{0}^{\infty} r^{2} d r \delta \varepsilon\left(\frac{1}{\gamma} f\right) \\
& =4 \pi \int d t \int_{0}^{\infty} r^{2} d r\left(\frac{\delta \varepsilon}{\delta n}\right)\left(-\frac{\delta f}{\gamma}+v \gamma f \delta v\right)
\end{aligned}
$$

and,

$$
\begin{gathered}
\delta v=-\frac{\delta f}{f} v-\frac{1}{r^{2} f} \int_{0}^{r} r^{2} \delta \dot{f} d r \\
\delta I=4 \pi \int d t \int_{0}^{\infty} r^{2} d r\left(\frac{\delta \varepsilon}{\delta n}\right)\left(-\frac{\delta f}{\gamma}+v \gamma\left\{-\delta f v-\frac{1}{r^{2}} \int_{0}^{r} r^{\prime 2} \delta \dot{f} d r^{\prime}\right\}\right) \\
=-4 \pi \int d t\left[\int_{0}^{\infty} r^{2} d r\left(\frac{\delta \varepsilon}{\delta n}\right) \gamma \delta f+\int_{0}^{\infty} d r\left(\frac{\delta \varepsilon}{\delta n}\right) v \gamma \int_{0}^{r} r^{\prime 2} \delta \dot{f} d r^{\prime}\right] \\
=-4 \pi \int d t \int_{0}^{\infty} r^{2} d r\left\{\left(\frac{\delta \varepsilon}{\delta n}\right) \gamma-\frac{\partial}{\partial t}\left[\int_{r}^{\infty} d r\left(\frac{\delta \varepsilon}{\delta n}\right) v \gamma\right]\right\} \delta f
\end{gathered}
$$

From this we have

$$
\left(\frac{\delta \varepsilon}{\delta n}\right) \gamma-\frac{\partial}{\partial t}\left[\int_{r}^{\infty} d r\left(\frac{\delta \varepsilon}{\delta n}\right) v \gamma\right]=0
$$

Taking the derivative with respect to $r$ of the both sides, we get

$$
\frac{\partial}{\partial t}\left[\left(\frac{\delta \varepsilon}{\delta n}\right) v \gamma\right]=-\frac{\partial}{\partial r}\left[\left(\frac{\delta \varepsilon}{\delta n}\right) \gamma\right]
$$

Using the adiabatic relation of the energy density and pressure in the equation of motion, we get

$$
\dot{v}+v \frac{\partial v}{\partial r}=-\frac{1}{(\varepsilon+P) \gamma^{2}}\left\{\frac{\partial P}{\partial r}+v \frac{\partial P}{\partial t}\right\}
$$

which is again the relativistic Euler equation for spherically symmetric case [27].

\section{A. Effective Lagrangian}

Now let us introduce a parametric ansatz for the density profile as

$$
\bar{n}(t, r)=f(r, a(t)),
$$

where $a=a(t)$ is a (set of) time-dependent parameter(s) which determines the form of $f$. We suppose that $f$ is suitably normalized,

$$
4 \pi \int_{0}^{\infty} r^{2} f d r=N
$$

The velocity profile, Eq.(31) becomes 


$$
v(r, t)=-\dot{a} \frac{1}{r^{2} f} \int_{0}^{r} r^{2}\left(\frac{\partial f}{\partial a}\right) d r \equiv \Delta \dot{a},
$$

with

$$
\Delta=\Delta(r, a)=-\frac{1}{r^{2} f} \int_{0}^{r} r^{2}\left(\frac{\partial f}{\partial a}\right) d r^{\prime} .
$$

For more than one parameter, $a=\left\{a^{i}, i=1, \ldots, n\right\}$ Eqs.(31) and (40) should be understood as

$$
v(r, t)=\sum_{i=1}^{n} \Delta_{i} \dot{a}^{i}, \Delta_{i}=-\frac{1}{r^{2} f} \int_{0}^{r} r^{\prime 2}\left(\frac{\partial f}{\partial a^{i}}\right) d r^{\prime} .
$$

The important point here is that the velocity field is linear in $\dot{a}$. The effective Lagrangian for our variable $a=a(t)$ becomes

$$
L(a, \dot{a})=-4 \pi \int_{0}^{\infty} r^{2} d r \varepsilon(n),
$$

where $n=f(r, a) / \gamma$ and $\gamma=1 / \sqrt{1-(\Delta \dot{a})^{2}}$. The equation of motion for the variable $a$ is obtained from the Euler-Lagrange equation

$$
\frac{d}{d t}\left(\frac{\partial L}{\partial \dot{a}}\right)-\frac{\partial L}{\partial a}=0
$$

which is written as

$$
4 \pi \int_{0}^{\infty} r^{2} d r\left[\frac{d}{d t}\left\{\frac{\delta \varepsilon}{\delta n} n \gamma^{2} \Delta^{2} \dot{a}\right\}+\frac{\delta \varepsilon}{\delta n} \frac{\partial n}{\partial a}\right]=0
$$

For adiabatic motion, we get

$$
\frac{d}{d t} \int_{0}^{\infty} r^{2} d r\left\{(\varepsilon+P) \gamma^{2} v \Delta\right\}=-\int_{0}^{\infty} r^{2} d r(\varepsilon+P)\left(\frac{1}{f} \frac{\partial f}{\partial a}-\gamma^{2} v \frac{\partial v}{\partial a}\right)
$$

The effective Hamiltonian $H$ is then

$$
\begin{aligned}
H & \equiv \dot{a} \frac{\partial L}{\partial \dot{a}}-L \\
& =4 \pi \int r^{2} d r\left[-\frac{\delta \varepsilon}{\delta n} \bar{n} \dot{a} \frac{\partial}{\partial \dot{a}}\left(\frac{1}{\gamma}\right)+\varepsilon\right]=4 \pi \int r^{2} d r\left[(\varepsilon+P) \gamma^{2} v^{2}+\varepsilon\right] \\
& =4 \pi \int r^{2} d r\left[(\varepsilon+P) \gamma^{2}-P\right]=\int d^{3} r T^{00}
\end{aligned}
$$

which is in fact the total energy of the system and a conserved quantity.

\section{B. Relativistic Rayleigh-Plesset Equation}

For an example, let us consider a system composed of a homogeneous spherical gas bubble surrounded by a homogeneous fluid. We then introduce the ansatz,

$$
\begin{aligned}
f & =\rho_{0 G}=\frac{3}{4 \pi} \frac{N_{G}}{R^{3}}, \quad 0<r<R \\
& =\rho_{0 L}=\frac{3}{4 \pi} \frac{N_{L}}{R_{\infty}^{3}-R^{3}}, \quad R<r<R_{\infty}
\end{aligned}
$$

where the radius of the gas bubble $R=R(t)$ is the only dynamic variable. We use the subscript $G$ and $L$ to specify the quantities in the gas and the fluid, respectively. For example, $N_{G}$ and $N_{L}$ are number of particles (constant) in 
the gas and fluid, respectively. The outer (constant) radius of the fluid $R_{\infty}$ is introduced here to take into account the conservation of the number of particles in the fluid, but ultimately should be taken equal to $\infty$. The velocity field is then determined as

$$
\begin{aligned}
v(r) & =\frac{r}{R} \dot{R}, \quad 0<r<R, \\
& =\frac{R^{2}\left(R_{\infty}^{3}-r^{3}\right)}{r^{2}\left(R_{\infty}^{3}-R^{3}\right)} \dot{R}, \quad R<r<R_{\infty},
\end{aligned}
$$

so that

$$
\begin{aligned}
\Delta(r) & =\frac{r}{R}, \quad 0<r<R, \\
& =\frac{R^{2}\left(R_{\infty}^{3}-r^{3}\right)}{r^{2}\left(R_{\infty}^{3}-R^{3}\right)}, \quad R<r<R_{\infty} .
\end{aligned}
$$

The effective Lagrangian for $R$ is then

$$
L=-4 \pi \int_{0}^{R} r^{2} d r \varepsilon_{G}\left(f_{G} \sqrt{1-\left(\frac{r}{R} \dot{R}\right)^{2}}\right)-4 \pi \int_{R}^{R_{\infty}} r^{2} d r \varepsilon_{L}\left(f_{L} \sqrt{1-\left(\frac{R^{2}\left(R_{\infty}^{3}-r^{3}\right)}{r^{2}\left(R_{\infty}^{3}-R^{3}\right)} \dot{R}\right)^{2}}\right)
$$

The equation of motion for $R=R(t)$ is given by

$$
\frac{d}{d t}\left(\frac{\partial L}{\partial \dot{R}}\right)=\frac{\partial L}{\partial R}
$$

where

$$
\frac{\partial L}{\partial \dot{R}}=4 \pi \frac{\dot{R}}{R^{2}} \int_{0}^{R} d r r^{4}\left(\varepsilon_{G}+P_{G}\right) \gamma^{2}+4 \pi R^{4} \dot{R} \int_{R}^{R_{\infty}} \frac{d r}{r^{2}}\left(\varepsilon_{L}+P_{L}\right) \gamma^{2}\left(\frac{R_{\infty}^{3}-r^{3}}{R_{\infty}^{3}-R^{3}}\right)^{2}
$$

and

$$
\begin{aligned}
\frac{\partial L}{\partial R}= & -4 \pi R^{2}\left[\varepsilon_{G}-\varepsilon_{L}\right]_{R}-4 \pi \int_{0}^{R} r^{2} d r\left(\varepsilon_{G}+P_{G}\right)\left(-\frac{3}{R}+\gamma^{2}\left(\frac{\dot{R}}{R}\right)^{2} \frac{r^{2}}{R}\right) \\
& -4 \pi \int_{R}^{R_{\infty}} r^{2} d r\left(\varepsilon_{L}+P_{L}\right)\left(\frac{3 R^{2}}{R_{\infty}^{3}-R^{3}}-\gamma^{2} \frac{R^{3}\left(2 R_{\infty}^{3}+R^{3}\right)\left(R_{\infty}^{3}-r^{3}\right)^{2}}{r^{4}\left(R_{\infty}^{3}-R^{3}\right)^{3}} \dot{R}^{2}\right) .
\end{aligned}
$$

At this stage, we can take the limit $R_{\infty} \rightarrow \infty$. Thus, we have

$$
\frac{\partial L}{\partial \dot{R}}=4 \pi \frac{\dot{R}}{R^{2}} \int_{0}^{R} d r r^{4}\left(\varepsilon_{G}+P_{G}\right) \gamma^{2}+4 \pi R^{4} \dot{R} \int_{R}^{\infty} \frac{d r}{r^{2}}\left(\varepsilon_{L}+P_{L}\right) \gamma^{2}
$$

and

$$
\begin{aligned}
\frac{\partial L}{\partial R}= & -4 \pi R^{2}\left(\left[\varepsilon_{G}-\varepsilon_{L}\right]_{R}+\left[\varepsilon_{L}+P_{L}\right]_{\infty}\right)+\frac{12 \pi}{R} \int_{0}^{R} r^{2} d r\left(\varepsilon_{G}+P_{G}\right) \\
& -4 \pi \int_{0}^{R} r^{2} d r\left(\varepsilon_{G}+P_{G}\right) \gamma^{2}\left(\frac{\dot{R}}{R}\right)^{2} \frac{r^{2}}{R}+8 \pi R^{3} \dot{R}^{2} \int_{R}^{\infty} \frac{d r}{r^{2}}\left(\varepsilon_{L}+P_{L}\right) \gamma^{2}
\end{aligned}
$$

The equation of motion then takes the form,

$$
\frac{d}{d t}\left[\left(I_{1}+I_{2}\right) R^{3} \dot{R}\right]=F-\left(I_{1}-2 I_{2}\right) R^{2} \dot{R}^{2},
$$


where

$$
\begin{aligned}
& I_{1}=\int_{0}^{1} d x x^{4}\left(\varepsilon_{G}+P_{G}\right) \gamma^{2}, \\
& I_{2}=\int_{1}^{\infty} \frac{d x}{x^{2}}\left(\varepsilon_{L}+P_{L}\right) \gamma^{2},
\end{aligned}
$$

with $x=r / R$ and

$$
F=-R^{2}\left(\left[\varepsilon_{G}-\varepsilon_{L}\right]_{R}+\left[\varepsilon_{L}+P_{L}\right]_{0}\right)+\frac{3}{R} \int_{0}^{R} r^{2} d r\left(\varepsilon_{G}+P_{G}\right)
$$

where the subscript 0 represents the quantity evaluated at $v=0$. Eq.(56) is a full relativistic equation of motion for the radius of a gas bubble under the homologous motion of the system.

To see the non-relativistic limit of Eq.(56), we separate the energy density into the sum of the rest-mass energy density $\rho$ and the internal energy density $\varepsilon^{i n t}$ as

$$
\varepsilon=\rho+\varepsilon^{i n t}
$$

and expand Eqs. (57,58,59) in a power series of small parameters such as $, v^{2}, \varepsilon_{\text {int }} / \rho$, and $P / \rho$ in the non-relativistic regime. We have

$$
(\varepsilon+P) \gamma^{2} \simeq \rho_{0}+\varepsilon_{0}^{i n t}+P_{0}+\frac{1}{2} \rho_{0} v^{2}
$$

Thus,

$$
\begin{aligned}
& I_{1} \simeq \frac{1}{5}\left(\rho_{0, G}+\varepsilon_{0, G}^{i n t}+P_{0, G}\right)+\frac{1}{14} \rho_{0, G} \dot{R}^{2} \\
& I_{2} \simeq \rho_{0, L}+\varepsilon_{0, L}^{i n t}+P_{0, L}+\frac{1}{10} \rho_{0, L} \dot{R}^{2}
\end{aligned}
$$

and

$$
\begin{aligned}
F \simeq & R^{2}\left(P_{0, G}-P_{0, L}\right)-\left(\frac{1}{2}\left(\rho_{0, L}+P_{0, L}\right)+\frac{3}{10}\left(\rho_{0, G}+P_{0, G}+c_{s}^{2} \rho_{0, G}\right)\right) R^{2} \dot{R}^{2} \\
& -\frac{1}{8}\left(\rho_{0, L}+\frac{3}{7} \rho_{G, 0}\right) R^{2} \dot{R}^{4} .
\end{aligned}
$$

In the lowest order in $\dot{R}$ we get

$$
\left(\frac{1}{5} \rho_{0, G}+\rho_{0, L}\right) R \ddot{R}+\frac{3}{2} \rho_{0, L} \dot{R}^{2}=P_{0, G}-P_{0, L},
$$

which is the usual Rayleigh-Plesset equation of a gas bubble inside a liquid [16] without the term for the energy dissipation due to the sound radiation.

When the equation of state of the fluid and the gas are given as

$$
P \propto \rho^{\Gamma}
$$

where $\Gamma$ is the adiabatic index, all the integrals in Eq.(56) are expressed analytically. We get

$$
\begin{aligned}
& M R^{3} \ddot{R}+\left\{\frac{1}{5} \rho_{0, G} J_{1}+\rho_{0, L} J_{3}+\frac{4-3 \Gamma_{G}}{5}\left(\varepsilon^{i n}+P\right)_{0, G} J_{2}+\left(\varepsilon^{i n}+P\right)_{0, L} J_{4}\right\} R^{2} \dot{R}^{2} \\
= & -R^{2}\left(\left[\varepsilon_{G}-\varepsilon_{L}\right]_{R}+\left[\varepsilon_{L}+P_{L}\right]_{0}\right)+R^{2}\left(\rho_{0, G} J_{9}+\left(\varepsilon^{i n}+P\right)_{0, G} J_{10}\right),
\end{aligned}
$$

with 


$$
\begin{aligned}
M= & \frac{1}{5} \rho_{0, G} J_{1}+\rho_{0, L} J_{3}+\frac{1}{5}\left(\varepsilon^{i n}+P\right)_{0, G} J_{2}+\left(\varepsilon^{i n}+P\right)_{0, L} J_{4} \\
& +\left[\frac{1}{7}\left(\rho_{0, G} J_{5}+(2-\Gamma)\left(\varepsilon^{i n}+P\right)_{0, G} J_{6}\right)+\frac{1}{5}\left(\rho_{0, L} J_{7}+(2-\Gamma)\left(\varepsilon^{i n}+P\right)_{0, L} J_{8}\right)\right] \dot{R}^{2}
\end{aligned}
$$

and

$$
\begin{aligned}
& J_{1}=F\left(\left[\frac{1}{2}, \frac{5}{2}\right], \frac{7}{2}, \dot{R}^{2}\right), J_{2}=F\left(\left[1-\frac{\Gamma}{2}, \frac{5}{2}\right], \frac{7}{2}, \dot{R}^{2}\right), \\
& J_{3}=F\left(\left[\frac{1}{2}, \frac{1}{4}\right], \frac{5}{4}, \dot{R}^{2}\right), J_{4}=F\left(\left[1-\frac{\Gamma}{2}, \frac{1}{4}\right], \frac{5}{4}, \dot{R}^{2}\right), \\
& J_{5}=F\left(\left[\frac{3}{2}, \frac{7}{2}\right], \frac{9}{2}, R^{2}\right), J_{6}=F\left(\left[2-\frac{\Gamma}{2}, \frac{7}{2}\right], \frac{9}{2}, \dot{R}^{2}\right), \\
& J_{7}=F\left(\left[\frac{3}{2}, \frac{5}{4}\right], \frac{9}{4}, \dot{R}^{2}\right), J_{8}=F\left(\left[2-\frac{\Gamma}{2}, \frac{5}{4}\right], \frac{9}{4}, \dot{R}^{2}\right), \\
& J_{9}=F\left(\left[\frac{3}{2},-\frac{1}{2}\right], \frac{5}{2}, \dot{R}^{2}\right), J_{10}=F\left(\left[\frac{3}{2},-\frac{\Gamma}{2}\right], \frac{5}{2}, \dot{R}^{2}\right),
\end{aligned}
$$

where $F([a, b], c, z)$ is the hypergeometric function.

For the sake of illustration, we show in Figs.1, 2, and 3, time dependences of the radius and velocity described by the relativistic Rayleigh-Plesset equation Eq. 67] for 3 different initial conditions. In this example, we consider the case where the both gas and fluid have the same mass density and the adiabatic index $\Gamma=4 / 3$. Three cases shown here are for the different values of the initial gas pressure, $(P / \rho)_{G, 0}=1 / 10$ (Fig. 1), 5 (Fig. 2), and 100 (Fig. 3), keeping the ratio of the initial gas to the liquid pressure $P_{G, 0} / P_{L, 0}=100$. Solid lines are for the full relativistic equation of motion (Eq.67) and the dashed ones are for the non-relativistic limit, Eq.(65). For the low initial gas pressure, two solutions coincide (Fig. 1). For the extremely high initial pressure, the motion of the bubble becomes completely relativistic (Fig. 3) and the non-relativistic equation of motion differs completely from the relativistic equation. Note that in the relativistic equation, the velocity saturates at $v / c=1$. Of course, in this extreme example, the fluid motion becomes supersonic $\left(v>v_{s}=\sqrt{1 / 3}=0.577\right)$ and the hypothesis of homologous motion may breakdown. However, it is important to note that, there exists a case where the non-relativistic approximation fails down completely although the fluid and gas motion are still subsonic like in the case of Fig.2.

\section{NONADIABATIC PROCESSES}

In many cases, the change of the density associated with the flow of the matter causes non-quasi static processes within the hydrodynamical volume element established in the practical calculations. For example, in the limit of large Reynolds number, the dynamical change of the volume easily leads to a highly turbulent regime in small regions of the fluid, and this complex fluid motion will gradually thermalize inside the volume element. In such a case, there appears the heat production inside of such a volume element. If the time scale for the thermalization is negligible, then the heat production can be expressed in terms of viscous tensor, and the hydrodynamic equation of motion becomes,

$$
\partial_{\mu}\left(T^{\mu \nu}+\Sigma^{\mu \nu}\right)=0
$$

where $\Sigma^{\mu \nu}$ is the shear tensor. When there is no heat transfer, we may take

$$
\Sigma^{\mu \nu}=q\left(u^{\mu} u^{\nu}-g^{\mu \nu}\right),
$$

where $q$ is a function of the local thermodynamical quantities, like $\rho, P$ and its derivatives. From Eq.(69) we have

$$
u^{\mu} \partial_{\mu}\left(\frac{\varepsilon}{n}\right)+(P+q) u^{\mu} \partial_{\mu}\left(\frac{1}{n}\right)=0 .
$$


This means that, in the Lagrange comoving system, the change of the specific energy with respect to the proper time is given by

$$
\frac{d E}{d \tau}+P \frac{d V}{d \tau}=-q \frac{d V}{d \tau}
$$

Thus we identify the function $q$ as the rate of the production of the entropy $S$ with respect to the volume change,

$$
q=-T \frac{d S}{d \tau} / \frac{d V}{d \tau}
$$

The specific form of $\Sigma^{\mu \nu}$, Eq.(70) allows us to write

$$
\tilde{T}^{\mu \nu}=(\varepsilon+P+q) u^{\mu} u^{\nu}-(P+q) g^{\mu \nu},
$$

which conserves

$$
\partial_{\mu} \tilde{T}^{\mu \nu}=0
$$

From the above conservation law we get immediately the equation of motion,

$$
\dot{v}+v \frac{\partial v}{\partial r}=-\frac{1}{(\varepsilon+P+q) \gamma^{2}}\left\{\frac{\partial(P+q)}{\partial r}+v \frac{\partial(P+q)}{\partial t}\right\}
$$

which describes the relativistic hydrodynamical motion under the local entropy production, Eq. (72). The function $q$ should be specified appropriately according to the non-adiabatic processes representing the conversion of kinetic energy of the collective motion to the internal energy of the matter. Such a viscosity was first introduced in the nonrelativistic hydrodynamics by Neumann and Richtmyer [17] in order to simulate the entropy production mechanism at the shock front. Eq. (76) is the relativistic extension of the method of pseudo-viscosity of Neumann and Richtmyer.

The above scheme is easily incorporated in the variational formalism. In the presence of non-adiabatic processes, the variation in the specific energy in the previous section, Eq. (18), should be replaced by

$$
\delta E=-P \delta V+\delta Q=-P \delta V+T \delta S=-(P+q) \delta V
$$

where $\delta Q$ is the generated heat associated with the non quasi-static density variation. Consequently we should, instead of the adiabatic relations $(19,20)$, use

$$
\begin{aligned}
\frac{\partial \varepsilon}{\partial n} & \rightarrow \frac{\varepsilon+P+q}{n}, \\
d\left(\frac{\delta \varepsilon}{\delta n}\right) & \rightarrow \frac{1}{n} d(P+q) .
\end{aligned}
$$

From these substitutions, we get immediately Eq. (76). In terms of parametric representation, the equation of motion is given by

$$
\frac{d}{d t} \int_{0}^{\infty} r^{2} d r\left\{(\varepsilon+P+q) \gamma^{2} v \Delta\right\}=-\int_{0}^{\infty} r^{2} d r(\varepsilon+P+q)\left(\frac{1}{f} \frac{\partial f}{\partial a}-\gamma^{2} v \frac{\partial v}{\partial a}\right)
$$

The effective Hamiltonian for dynamical variable $a$ is given again by

$$
\begin{aligned}
H & \equiv \dot{a} \frac{\partial L}{\partial \dot{a}}-L \\
& =4 \pi \int r^{2} d r\left[(\varepsilon+P+q) \gamma^{2}-(P+q)\right]=\int d^{3} r\left(T^{00}+\Sigma^{00}\right)
\end{aligned}
$$

which is conserved,

$$
\frac{d H}{d t}=0
$$

for the equation of motion, Eq.(30), together with Eq.(72). This $H$ can again be identified as the total energy of the system including the internal heat energy generated in the fluid. 


\section{GENERAL RELATIVISTIC HYDRODYNAMICS}

For the application of the present formalism to astrophysical problems it is essential to include the effect of gravity through the theory of General Relativity. The variational approach of the general relativistic hydrodynamics has been discussed by several authors 18 21. In this section, starting from the variational approach, we show that the method of effective Lagrangian can also be established taking the metric as one of the variational trial functions. Let us first review how the general relativistic energy and momentum tensor are derived from the variational approach.

\section{A. Energy-Momentum Tensor and Einstein's Equation}

The total action is given as

$$
I=I_{G}+I_{M}
$$

where

$$
I_{G}=\frac{1}{2 \kappa} \int d^{4} x \sqrt{-g} \Re,
$$

is the action for the gravitational field and $\kappa=8 \pi G$ with $G$ the gravitational constant. As usual, $g=$ det $\left|g_{\mu \nu}\right|$, is the determinant of the metric tensor $g_{\mu \nu}$, and $\Re$ is the curvature scalar. The action of matter is now given by 18

$$
I_{M}=\int d^{4} x \sqrt{-g}\left\{-\varepsilon(n)+\xi(x)\left(n u^{\mu}\right)_{; \mu}+\frac{1}{2} \zeta(x)\left(u^{\mu} u_{\mu}-1\right)\right\},
$$

where $\xi$ and $\zeta / 2$ are Lagrange multipliers as before. As usual, ";" represents the covariant derivative and the factor $\sqrt{-g}$ is inserted to guarantee that the Lagrangian density is a scalar. The variation of the action should be carried out with respect to $g_{\mu \nu}, n, u^{\mu}, \xi$, and $\zeta$, independently. The results of variations with respect to $n, u^{\mu}, \xi$, and $\zeta$ are the same as before (see Eqs.(12), (13), (14), (15), (29) and the comments for the covariant derivative in Sec.II). Thus, these variations gives the relativistic hydrodynamic equation for a given metric $g^{\mu \nu}$.

The functional derivative with respect to $g_{\mu \nu}$ is calculated to be

$$
\frac{\delta I_{M}}{\delta g_{\mu \nu}}=-\frac{\partial \sqrt{-g}}{\partial g_{\mu \nu}}\left\{\varepsilon(n)+n\left(\partial_{\mu} \xi(x)\right) u^{\mu}\right\}+\frac{1}{2} \zeta \sqrt{-g} u^{\mu} u^{\nu},
$$

where we already employed the constraints, cf. Eqs.(4, (4). Using

$$
\frac{\partial \sqrt{-g}}{\partial g_{\mu \nu}}=-\frac{1}{2} \sqrt{-g} g^{\mu \nu}
$$

and substituting the values of $\partial_{\mu} \xi$ and $\zeta$, we get,

$$
\begin{aligned}
\frac{\delta I_{M}}{\delta g_{\mu \nu}} & =\frac{1}{2} \sqrt{-g} g^{\mu \nu}\left\{\varepsilon(n)-n\left(\frac{\varepsilon+P}{n} u_{\mu}\right) u^{\mu}\right\}+\frac{1}{2} \sqrt{-g}(\varepsilon+P) u^{\mu} u^{\nu} \\
& =\frac{1}{2} \sqrt{-g}\left\{(\varepsilon+P) u^{\mu} u^{\nu}-P g^{\mu \nu}\right\} .
\end{aligned}
$$

Comparing this result to the definition of the energy-momentum tensor,

$$
\frac{\delta I_{M}}{\delta g_{\mu \nu}} \equiv \frac{1}{2} \sqrt{-g} T^{\mu \nu}
$$

we identify that

$$
T^{\mu \nu}=(\varepsilon+P) u^{\mu} u^{\nu}-P g^{\mu \nu},
$$

which is nothing but the energy-momentum tensor of the perfect fluid. Thus, the energy-momentum tensor of the fluid is derived from the Lagrangian density Eq.(84) just as in the case of the field theoretical Lagrangian. Note that the role of constraints are essential for this derivation. 
The variation of the gravitational action $I_{G}$ with respect to $g_{\mu \nu}$ gives the usual Einstein tensor $\mathcal{G}^{\mu \nu}$,

$$
\frac{\delta I_{G}}{\delta g_{\mu \nu}} \equiv-\frac{1}{2} \sqrt{-g} \mathcal{G}^{\mu \nu}
$$

so that we get

$$
\mathcal{G}^{\mu \nu}=\kappa T^{\mu \nu},
$$

which is the Einstein equation, as expected. The hydrodynamical equation, Eq.(29),

$$
T_{; \mu}^{\mu \nu}=0,
$$

can be re-obtained from Eq.(90) due to the Bianchi identity,

$$
\mathcal{G}_{; \mu}^{\mu \nu}=0
$$

It is interesting to note that if we use the metric functions as basic dynamical variables then, the hydrodynamic equation of motion is obtained somewhat indirectly from the properties of metric tensor and the variational principle does not lead directly to the equation of motion. This point will be discussed later again in the context of the derivation of the special relativistic equation of motion using the comoving coordinate system.

\section{B. Spherically Symmetric System}

The derivation of the equation of motion above is too formal and not much useful to be applied directly for some practical problems. To make use of the variational approach, it is necessary to establish appropriate trial functions in order to write down the effective Lagrangian for these functions. As in the case of special relativity, this is possible when the system has appropriate symmetry, such as spherically symmetric distribution of matter. Many problems of the gravitational collapse of stars, the structure of neutron stars, and the Robertson-Walker cosmology can be discussed in this symmetry. Here we establish the effective Lagrangian for the spherically symmetric system.

The most general form of the metric for a spherically symmetric system can be taken as 27.

$$
d s^{2}=e^{2 \phi} d T^{2}-e^{2 \lambda} d \xi^{2}-r^{2} d \Omega^{2},
$$

where $(T, \xi)$ denotes the time and radial coordinates and $\phi=\phi(\xi, T), \lambda=\lambda(\xi, T)$, and $r=r(\xi, T)$ are unknown functions to be determined. Usually, if we consider the radial velocity field of the fluid as an independent variable, then we need only two independent functions in the metric and we may choose, for example, $r=\xi$. However, with the above metric involving three functions, we can further take the so-called comoving frame in such a way that the space-like components of the four-velocity field vanish everywhere 28,

$$
u^{\mu}=\left(u^{0}, 0,0,0\right) .
$$

From the normalization condition $u_{\mu} u^{\mu}=1$, we get

$$
u^{0}=e^{-\phi}
$$

In this comoving frame, the conservation law is expressed as

$$
\left(n u^{\mu}\right)_{; \mu}=\frac{1}{\sqrt{-g}} \partial_{\mu}\left(\sqrt{-g} n u^{\mu}\right)=\frac{1}{r^{2} e^{\lambda} e^{\phi}} \partial_{T}\left(r^{2} e^{\lambda} n\right)=0
$$

so that the density $n$ of the conserved quantity, say the baryon number, is given by

$$
n=\frac{\rho}{e^{\lambda} r^{2}},
$$

where $\rho=\rho(\xi)$ should be determined by the initial condition. In this choice of the metric, the matter Lagrangian density is expressed as

$$
\mathcal{L}_{M}=-\sqrt{-g} \varepsilon(n)=-e^{\phi} e^{\lambda} r^{2} \varepsilon(n),
$$


where $n$ is given by Eq.997). No terms with Lagrangian multipliers appear, because the constraints are automatically satisfied. The gravitational part is calculated as

$$
\mathcal{L}_{G}=\frac{1}{2 \kappa} e^{\lambda} e^{\phi}\left[r^{\prime}\left(2 r \phi^{\prime}+r^{\prime}\right) e^{-2 \lambda}-\dot{r}(2 r \dot{\lambda}+\dot{r}) e^{-2 \phi}+1\right],
$$

where we introduced the notation $\dot{f}=\partial f / \partial T$ and $f^{\prime}=\partial f / \partial \xi$. In the above, we omitted the part which can be written as the total derivative of a function, since this does not alter the equation of motion. The total Lagrangian of the spherically symmetric system is then given explicitly as

$$
\mathcal{L}[\phi, \lambda, r]=e^{\phi} e^{\lambda} r^{2}\left\{-\varepsilon(n)+\frac{1}{2 \kappa r^{2}}\left[r^{\prime}\left(2 r \phi^{\prime}+r^{\prime}\right) e^{-2 \lambda}-\dot{r}(2 r \dot{\lambda}+\dot{r}) e^{-2 \phi}+1\right]\right\} .
$$

When the variation for functions $\phi, \lambda$, and $r$ are in fact arbitrary, this Lagrangian is equivalent to Einstein's equations. To see this, we write the Euler-Lagrange equations of motion for $\phi, \lambda$, and $r$ from this Lagrangian to get

$$
\begin{gathered}
\varepsilon=\frac{1}{\kappa}\left[\frac{1}{r^{2}}+2 e^{-2 \lambda}\left(\frac{r^{\prime}}{r} \lambda^{\prime}-\frac{r^{\prime \prime}}{r}-\frac{r^{\prime 2}}{2 r^{2}}\right)+2 e^{-2 \phi}\left(\frac{\dot{r}^{2}}{2 r^{2}}+\frac{\dot{r}}{r} \dot{\lambda}\right)\right], \\
P=-\frac{1}{\kappa}\left[\frac{1}{r^{2}}-2 e^{-2 \lambda}\left(\frac{r^{\prime}}{r} \phi^{\prime}+\frac{r^{\prime 2}}{2 r^{2}}\right)+2 e^{-2 \phi}\left(\frac{\ddot{r}}{r}+\frac{\dot{r}^{2}}{2 r^{2}}-\frac{\dot{r}}{r} \dot{\phi}\right)\right],
\end{gathered}
$$

and

$$
\begin{aligned}
P= & -\frac{1}{\kappa}\left[-e^{-2 \lambda}\left(\phi^{\prime \prime}+\phi^{2}-\phi^{\prime} \lambda^{\prime}+\frac{1}{r}\left(r^{\prime \prime}+\phi^{\prime} r^{\prime}-\lambda^{\prime} r^{\prime}\right)\right)\right. \\
& \left.+e^{2 \phi}\left(\ddot{\lambda}+\dot{\lambda}^{2}-\dot{\phi} \dot{\lambda}+\frac{1}{r}(\ddot{r}+\dot{r} \dot{\lambda}-\dot{\phi} \dot{r})\right)\right] .
\end{aligned}
$$

We verify directly that these three equations are exactly those corresponding to the diagonal part of Einstein's equation. In fact, writing these equations in the form

$$
\begin{gathered}
\varepsilon=\frac{1}{\kappa} \mathcal{G}_{0}^{0}, \\
P=-\frac{1}{\kappa} \mathcal{G}_{1}^{1},
\end{gathered}
$$

and

$$
P=-\frac{1}{\kappa} \mathcal{G}_{2}^{2},
$$

where $\mathcal{G}_{0}^{0} \mathcal{G}_{1}^{1}$ and $\mathcal{G}_{2}^{2}$ 's are defined, respectively, as the quantities in the square bracket [ ] of Eqs.(101), (102), and (103), we can identify the functions $\mathcal{G}$ as the diagonal components of the Einstein tensor corresponding to the metric (93).

The only difference between our formalism here and Einstein's equation is that in the former there is no equation corresponding to the non-diagonal element, $\mathcal{G}_{1}^{0}$ in the latter. In Einstein's theory, this quantity should be zero,

$$
\mathcal{G}_{1}^{0}=\frac{2 e^{-2 \lambda}}{r}\left(\dot{r}^{\prime}-\dot{r} \phi^{\prime}-\dot{\lambda} r^{\prime}\right)=0 .
$$

This is because in the comoving frame the energy-momentum tensor $T_{\nu}^{\mu}$ is diagonal. Therefore, to prove that our result is identical to the usual theory, we have to show that Eq.(107) is a consequence of Eqs.(104) - 106). Although this proof is rather basic matter and could be found in text books, we show it explicitly for the sake of later discussion. We first start with the well-known Bianchi identity (for example, see [27], p.363),

$$
\mathcal{G}_{\nu ; \mu}^{\mu}=\partial_{\mu}\left(\sqrt{-g} \mathcal{G}^{\mu}\right)+\frac{1}{2} \sqrt{-g}\left[\mathcal{G}_{\alpha \beta} \partial_{\nu} g^{\alpha \beta}\right]=0 .
$$


In our case, the first component $\nu=0$ leads to

$$
\partial_{0}\left(\sqrt{-g} \mathcal{G}_{0}^{0}\right)+\partial_{1}\left(\sqrt{-g} \mathcal{G}_{0}^{1}\right)+\frac{1}{2} \sqrt{-g}\left[\sum_{\alpha=0}^{3} g_{\alpha \alpha} \mathcal{G}^{\alpha}{ }_{\alpha} \partial_{0} g^{\alpha \alpha}\right]=0
$$

On the other hand, we have

$$
\begin{aligned}
& \partial_{0}\left(\sqrt{-g} \mathcal{G}_{0}^{0}\right)+\frac{1}{2} \sqrt{-g}\left[\sum_{\alpha=0}^{3} g_{\alpha \alpha} \mathcal{G}_{\alpha}^{\alpha} \partial_{0} g^{\alpha \alpha}\right] \\
= & r^{2} e^{\phi} e^{\kappa}\left\{\frac{\partial \mathcal{G}_{0}^{0}}{\partial t}+2 \frac{\dot{r}}{r}\left(\mathcal{G}_{2}^{2}-\mathcal{G}_{1}^{1}\right)+\left(\mathcal{G}_{0}^{0}+\mathcal{G}_{1}^{1}\right)\left(2 \frac{\dot{r}}{r}+\dot{\lambda}\right)\right\} \\
= & r^{2} e^{\phi} e^{\kappa}\left[\dot{\varepsilon}+(\varepsilon+p)\left(2 \frac{\dot{r}}{r}+\dot{\lambda}\right)\right]=0,
\end{aligned}
$$

where Eqs.104 - (106) together with the energy conservation,

$$
\dot{\varepsilon}=\frac{d \varepsilon}{d n} \frac{\partial\left(\rho / r^{2} e^{\lambda}\right)}{\partial T}=-(\varepsilon+P)\left(2 \frac{\dot{r}}{r}+\dot{\lambda}\right)
$$

are used. Comparing Eqs. (109) and (110), we get

$$
\partial_{1}\left(\sqrt{-g} \mathcal{G}_{0}^{1}\right)=0
$$

or

$$
r^{2} e^{\phi} e^{\lambda} \mathcal{G}_{0}^{1}=C(T) .
$$

where $C$ is a function of $T$ only. For a non-singular metric we should have $r(\xi=0, T)=0$, hence we conclude that $C(T) \equiv 0$. Therefore, we obtain

$$
\mathcal{G}_{0}^{1}=0
$$

This completes the proof that our result is equivalent to Einstein's equation. That is, the Lagrangian density Eq. (100) describes correctly the dynamics of a spherically symmetric system of an ideal fluid and gravitational field.

\section{Misner-Sharp Equation}

Together with Eq.(107), Eq.(113) implies the following relation,

$$
\dot{r}^{\prime}-\dot{r} \phi^{\prime}-\dot{\lambda} r^{\prime}=0,
$$

which can be obtained from Eqs.104 - (106) directly, without referring to the Einstein tensor $G_{\nu}^{\mu}$ and its properties.

Following Ref. 22] we can express the equations of motion in a more convenient form. First, putting $G_{1}^{0} \equiv 0$ in the second component of the Bianchi identity, we have

$$
\frac{\partial \mathcal{G}_{1}^{1}}{\partial r}+2 \frac{r^{\prime}}{r}\left(\mathcal{G}_{1}^{1}-\mathcal{G}_{2}^{2}\right)=-\left(\mathcal{G}_{0}^{0}+\mathcal{G}_{1}^{1}\right) \phi^{\prime}
$$

Substituting Eqs.(104) - (106), we get immediately that

$$
P^{\prime}=-(\varepsilon+P) \phi^{\prime},
$$

which is the Euler equation in the comoving frame. Now we introduce a quantity $U$ defined by

$$
U=\dot{r} e^{-\phi}=\frac{d r}{d \tau}
$$

where $\tau$ is the local proper time and $d / d \tau$ is the total derivative. The relation (114) is expressed in terms of $U$ as 


$$
e^{-\phi} \dot{\lambda}=\frac{U^{\prime}}{r^{\prime}}
$$

Now Eq.101) becomes

$$
8 \pi G \varepsilon r^{2}=1-r r^{\prime}\left(e^{-2 \lambda}\right)^{\prime}-e^{-2 \lambda}\left(r^{\prime \prime} R+r^{\prime 2}\right)+U^{2}+2 r U U^{\prime} r^{\prime-1},
$$

which can be integrated as

$$
e^{-2 \lambda}=\frac{1}{r^{\prime 2}}\left(1+U^{2}-\frac{2 M G}{r}\right)
$$

where, as before, $G$ is the gravitational constant and

$$
M(\xi, T)=4 \pi \int_{0}^{\xi} \varepsilon r^{2} r^{\prime} d \xi=4 \pi \int_{0}^{r} \varepsilon r^{2} d r
$$

Inserting Eq.(119) into (105), together with (116), and after some manipulations we obtain:

$$
\frac{d^{2} r}{d \tau^{2}}=\dot{U} e^{-\phi}=-\left(\frac{1}{\varepsilon+P}\right)\left(1+U^{2}-\frac{2 M G}{r}\right)\left(\frac{\partial P}{\partial r}\right)_{T}-G \frac{M+4 \pi R^{3} P}{r^{2}} .
$$

This form of the equation of motion was first obtained by Misner and Sharp [22]. Equations (116), (117), (120) and (121) together with an equation of state completely determine the dynamics of a spherical collapse, or bounce, which might be relevant for the study of the gamma ray bursts [10].

To see the relation between this expression and the special relativistic Euler equation Eq. (36), we set $G=0$ in Eq.(121),

$$
\frac{d^{2} r}{d \tau^{2}}=-\left(\frac{1}{\varepsilon+P}\right)\left(1+U^{2}\right)\left(\frac{\partial P}{\partial r}\right)_{T} .
$$

Let $(t, r)$ the coordinate system fixed in the Minkowskian space-time. Thus, the line element is given as

$$
d s^{2}=d t^{2}-d r^{2}-r^{2} d \Omega^{2},
$$

in a space-fixed coordinate system. However, in the comoving coordinate system, we need non-trivial metric functions as follows. First we introduce a coordinate transformation,

$$
\begin{aligned}
& r=r(T, \xi), \\
& t=t(T, \xi),
\end{aligned}
$$

where we may identify the coordinate $\xi$ as the comoving Lagrangian coordinate. Thus, by definition,

$$
v=\left(\frac{\partial r}{\partial t}\right)_{\xi=c o n t .} .
$$

It is always possible to choose the variable $T$ so that the cross term in the above equation vanishes and the line element can be written in the form of Eq.(93),

$$
d s^{2}=e^{2 \phi} d T^{2}-e^{2 \lambda} d \xi^{2}-r^{2}(\xi, t) d \Omega^{2} .
$$

Here,

$$
d \tau=\left.e^{\phi} d T\right|_{d \xi=0},
$$

is the (local) proper time. Note that a local Lorentz transformation relates the infinitesimal coordinate differences to those of proper time $d \tau$ and local radial distance $e^{\lambda} d \xi$ by

$$
\left(\begin{array}{c}
d t \\
d r
\end{array}\right)=\left(\begin{array}{ll}
\gamma & v \gamma \\
v \gamma & \gamma
\end{array}\right)\left(\begin{array}{c}
d \tau \\
e^{\lambda} d \xi
\end{array}\right)
$$


On the other hand, since

$$
\left.d s^{2}\right|_{d \xi=0}=d \tau^{2}=\left[d t^{2}-d r^{2}\right]_{d \xi=0}=\left.d t\right|_{d \xi=0} ^{2}\left(1-v^{2}\right)
$$

we conclude

$$
\left.\gamma^{-1} d t\right|_{d \xi=0}=d \tau=e^{\phi} d T
$$

In this way we have

$$
\begin{aligned}
\frac{d^{2} r}{d \tau^{2}} & \equiv e^{-\phi} \frac{\partial}{\partial T}\left(e^{-\phi} \frac{\partial r}{\partial T}\right)_{\xi}=\gamma \frac{\partial}{\partial t}\left(\gamma \frac{\partial r}{\partial t}\right)_{\xi} \\
& =\gamma\left(\frac{\partial \gamma}{\partial t} v+\gamma\left(\frac{\partial v}{\partial t}\right)_{\xi}\right) \\
& =\gamma^{2}\left(1+\gamma^{2} v^{2}\right)\left(\frac{\partial v}{\partial t}\right)_{\xi} .
\end{aligned}
$$

Now, from Eq.(126) we have

$$
\begin{aligned}
\left.d t\right|_{d T=0} & =v \gamma e^{\lambda} d \xi, \\
\left.d r\right|_{d T=0} & =\gamma e^{\lambda} d \xi
\end{aligned}
$$

so that

$$
\begin{aligned}
\left(\frac{\partial P}{\partial R}\right)_{T} & =\left(\frac{\partial P}{\partial r}\right)_{t}+\left(\frac{\partial P}{\partial t}\right)_{r}\left(\frac{\partial t}{\partial r}\right)_{T} \\
& =\left(\frac{\partial P}{\partial R}\right)_{t}+v\left(\frac{\partial P}{\partial t}\right)_{r}
\end{aligned}
$$

We also have

$$
\left(\frac{\partial v}{\partial t}\right)_{\xi}=\left(\frac{\partial v}{\partial t}\right)_{r}+\left(\frac{\partial v}{\partial r}\right)_{t}\left(\frac{\partial r}{\partial t}\right)_{\xi}=\left(\frac{\partial v}{\partial t}\right)_{r}+v\left(\frac{\partial v}{\partial r}\right)_{t} .
$$

Therefore, Eq.(121) becomes

$$
\left(\frac{\partial v}{\partial t}\right)_{r}+v\left(\frac{\partial v}{\partial r}\right)_{t}=-\frac{1}{\varepsilon+P} \frac{1}{\gamma^{2}}\left[\left(\frac{\partial P}{\partial r}\right)_{t}+v\left(\frac{\partial P}{\partial t}\right)_{r}\right]
$$

which is exactly the relativistic Euler equation (36).

\section{Variational Principle in Comoving Coordinate for No Gravity Limit $(G \rightarrow 0)$}

The above discussion suggests the possible use of the comoving (Lagrangian) coordinate system even for cases with no gravity, that is $G \rightarrow 0$. The effective Lagrangian presented in Sec.III is based on an ansatz for the solution of the continuity equation in the space-fixed coordinate system. By using a comoving Lagrange coordinate system, we may better choose the trial function on physical grounds. Of course, the two systems of coordinates, in principle, should be equivalent if the ansatz has enough flexibility to the express any arbitrary flow pattern of the matter. However, for practical applications, the appropriate choice of the coordinate system is essential to get better results. For example, it is technically difficult to introduce the velocity dependence in the ansatz for the density profile consistent with the continuity equation. Therefore, for an ansatz like Eq.(37), established in the space-fixed coordinate system, the relativistic kinematical effects may induce some spurious effects on the dynamics of the parameters. On the other hand, if we can choose the parametrization in the comoving coordinate system, such kinematical effects are expected to be automatically included in the equation of motion.

In the limit of $G \rightarrow 0$, the space-time reduces to that of Minkowski and obviously the gravitational part of the Lagrangian density (100) vanishes. However, in the comoving frame the line element has still the form (93) and 
functions $e^{\phi}$ and $e^{\lambda}$ remain unknown. If we drop out the gravitational part from the action, the variational principle does not give information on these functions. What should be done in this limit is that Eqs.(116) and (119) are used as constraints among the unknown functions, $r, \phi$ and $\lambda$. Setting $G=0$ in Eq.(119) and using the relation

$$
\sqrt{1+U^{2}}=\sqrt{1+\left(\dot{r} e^{-\phi}\right)^{2}}=\sqrt{1+\gamma^{2} v^{2}}=\gamma
$$

we obtain

$$
e^{\lambda}=\frac{r^{\prime}}{\gamma}
$$

On the other hand, assuming the isentropic initial condition and adiabatic process, we can integrate Eq.(116) with respect to $\xi$ to get

$$
e^{-\phi}=\frac{\varepsilon+P}{m n} \equiv h(n)
$$

where $h$ is the specific enthalpy of the matter and $m$ is the rest mass of the constituent particles. This integration constant was chosen so that $e^{\phi} \rightarrow 1, n \rightarrow 0$.

Now the action becomes

$$
I=-\int d T \int d \xi e^{\phi} e^{\lambda} r^{2} \varepsilon(n)=-\int d T \int d \xi \frac{r^{\prime} r^{2}}{\gamma} \frac{m n}{\varepsilon+P} \varepsilon(n),
$$

where

$$
n=\frac{\gamma \rho(\xi)}{r^{\prime} r^{2}}
$$

and $\rho=\rho(\xi)$ is determined from the initial condition.

Let the $T$-dependence of $r$ be specified as

$$
r(\xi, t)=f(\xi, a(T))
$$

Then the Lorentz factor $\gamma$ is expressed as

$$
\gamma=\sqrt{1+e^{-2 \phi} \dot{r}^{2}}=\sqrt{1+h^{2}(n)\left(\frac{\partial f}{\partial a}\right)^{2} \dot{a}^{2}},
$$

so that the number density $n=n(a, \dot{a} ; \xi)$ should be determined by the equation

$$
f^{\prime} f^{2} n=\rho(\xi) \sqrt{1+h^{2}(n)\left(\frac{\partial f}{\partial a}\right)^{2} \dot{a}^{2}} .
$$

Finally the effective Lagrangian for $a=a(T)$ is given by

$$
L(a, \dot{a})=-\int d \xi \rho(\xi) \varepsilon(n) .
$$

The Euler-Lagrange equation of motion for $a$ then takes the form

$$
\frac{d}{d t} \int d \xi \rho(\xi)(\varepsilon+P) \frac{1}{n}\left(\frac{\partial n}{\partial \dot{a}}\right)=\int d \xi \rho(\xi)(\varepsilon+P) \frac{1}{n}\left(\frac{\partial n}{\partial a}\right) .
$$

Differently from the case of the space-fixed coordinate system, we need the equation of state to determine the density of the matter as a function of our dynamical variable, (136). Note the difference between the density profiles $n(r, t)$ of the Sec.III and $n(\xi, T)$ defined by Eq.(137). The former is defined for constant time, $t=$ const. of the space fixed global coordinate system, and the latter is defined for constant time coordinate, $T=$ const. of the comoving coordinate system. 
Although the comoving coordinate system seems physically more advantageous than the space fixed global coordinate system in choosing an ansatz, it may generate a difficulty in solving Eq.(137) for a given equation of state. For the ideal gas, like

$$
P \propto n^{\gamma}
$$

with $\gamma=5 / 3$ or $4 / 3$, an analytic solution of Eq.(137) for $n$ can be obtained explicitly. However, for general cases, analytic solution is not available. To avoid this, one may be tempted to use the proper time defined by

$$
d \tau=e^{\phi} d T
$$

instead of the time coordinate $T$ and introduce the ansatz,

$$
r(\xi, \tau)=f(\xi, a(\tau)),
$$

in substitution for Eq.(136). In this case, the Lorentz factor becomes

$$
\gamma=\sqrt{1+\left(\frac{\partial f}{\partial a}\right)^{2}\left(\frac{d a}{d \tau}\right)^{2}}
$$

involving no density dependent term in it. Thus the density is expressed directly as

$$
n=\frac{\rho(\xi)}{f^{\prime} f^{2}} \sqrt{1+\left(\frac{\partial f}{\partial a}\right)^{2}\left(\frac{d a}{d \tau}\right)^{2}}
$$

without need for solving the equation, (137). However, unfortunately, the pair of variables, $(\tau, \xi)$ do not constitute the proper integrable coordinate system, so that the boundary condition for the variation principle on the action,

$$
I=-\int d \tau \int d \xi e^{\lambda} r^{2} \varepsilon(n)
$$

is not properly defined and a simple Euler-Lagrange equation for fixed $\tau$ leads to a wrong result.

\section{DISCUSSION AND CONCLUDING REMARKS}

The variational approach for systems of fields, including the general theory of relativity, is of course a basic and standard theoretical framework and has been well studied, even for the application to the hydrodynamics discussed here. However, to the authors' knowledge, except for the formal derivation, no explicit variational formulation for the practical application for relativistic hydrodynamical systems has ever been carried out.

From the formal point of view, matter described by hydrodynamics is rather a phenomenological concept than the consideration of the fundamental degrees of freedom. In field theories, the variational approach is indispensable in discussing, for example, the underlying symmetries of the matter field, such as Noether's theorem, the quantization procedure, etc. Most of these formal aspects of the variational approach will not be much useful for hydrodynamical systems, except for the obvious symmetries required for the energy-momentum tensor. Thus one might find no point in discussing hydrodynamics from the action principle, once the equations of motion of hydrodynamics are well established in terms of the equations for the energy-momentum tensor.

On the other hand, as is well-known, the variational approach has practical advantages besides its formal side. Once the variational principle is established, we can use the method to obtain the optimal parameters of a given family of trial solutions.

In this paper, we derived the equations of motion of hydrodynamics starting from a very simple Lagrangian density. There it is seen that the roles of the continuity equation as a dynamical constraint and of the local thermodynamical relations are essential to arrive at the standard result of hydrodynamics. When the continuity equation is soluble, such a formulation in terms of the variational principle offers a powerful tool to obtain approximate solutions. For a system with a high degree of symmetry such as spherically symmetric system, we can establish the effective Lagrangian for the density profile function. Such an effective Lagrangian is quite useful for obtaining the approximate solutions for the hydrodynamical equation of motion in a simple manner. Even for the finite element discretisation of the hydrodynamic equation designed to a larger numerical solution, the variational approach may offer a physically optimized equation of motion, avoiding the mathematical instability with a relatively small number of degrees of freedom [13. As an 
example of extreme simplified case, we apply the effective Lagrangian formulation for a gas bubble in a fluid and for the first time the relativistic version of the Rayleigh-Plesset equation is obtained. Such an approach will be useful for the analysis of the relativistic motions of blast waves in the models of gamma ray bursters [10], or the hot and dense droplet of QGP plasma, possibly formed in high-energy nuclear collisions. The application of our formalism for such processes is being planned. We have discussed also the introduction of pseudo-viscosity due to Neumann and Ritchmyer in the context of variational formulation. This will allow, for example, not only to treat relativistically the propagation of shock waves but also to introduce the finite relaxation time of turbulent flows in a phenomenological manner [14] in the relativistic fluid dynamics.

Our formalism will be useful in studying some problems of General Relativity, too. For a spherically symmetric system, a very simple Lagrangian density has been found. From this Lagrangian density we can show that all the known equations of the spherically symmetric system can be derived. We expect that, together with a good parametrization of the metric functions, approximate solutions to these otherwise difficult problems of stellar collapse or explosion with realistic equations of state can be obtained. Work on this line is in progress.

Authors wish to express their thanks to Drs. C.E.Aguiar and M.O.Calvão for enlightening discussion and critical comments. This work is supported in part by PRONEX (contract no. 41.96.0886.00), FAPESP(contract no. 98/22494) and CNPq-Brasil Processes, 300962/86-0, 573846/1997-9, 142338/97-4 and also by US-Department of Energy under Grant No. DE-FG03-95 ER40937, by NSF under grant INT-9602920.

[1] E. Fermi, Prog. Theor. Phys. 5, 570 (1950); Phys. Rev. 81 (1951), 683.

[2] L. D. Landau, Izv. Akad. Nauk SSSR, Ser. fiz., 17 (1953), 51; S. Z. Belenkij and L. D. Landau, N.Cim., Suppl., 3 (1956), 15.

[3] H. Stöcker and W. Greiner, Phys. Rep. 137 (1986) 277.

[4] R.B. Clare and D. Strottman, Phys. Rep. 141 (1986), 177 .

[5] L. P. Csernai: Introduction to Relativistic Heavy Ion Collisions, John Wiley \& Sons, New York, 1994.

[6] H.-Th. Elze and U. Heinz, Phys. Rep. 183 (1989), 81.

[7] P. J. E. Peebles, Physical Cosmology, Princeton Univ. Press, 1971.

[8] S. L. Shapiro, S. A. Teukolsky, Black Holes, White Dwarfs, and Neutron Stars, John Wiley \& Sons, New York, 1983.

[9] N. K. Glendenning, Compact Stars, Springer Verlach, 1995, H. A. Bethe, Rev. Mod. Phys. 62 (1990), 801.

[10] for example, G. J. Fishman and C. A. Meegan, Annu. Rev. Astron. Astrophys. 33 (1995), 415; C. Kouveliotou, Science, 277 (1997), 1257; B. Paczynski and C. Kouveliotou, Nature, 389 (1997), 548.

[11] for example, A. Mezzacappa and S. W. Bruenn, ApJ. 405 (1993), 669; ApJ.410 (1993), 740.

[12] for example, J. Rafelski, J. Letessier and A. Tounsi, in Relativistic Aspects of Nuclear Physics (T.Kodama et al, Ed.), Vol. 4, p.211, World Scientific Pub., Singapore, 1996.

[13] H. A. R. Gonçalves, S. B. Duarte, T. Kodama and V. D'Avila, Astron. \& Space Science, 194, 313 (1992).

[14] T. Kodama, R. Donangelo and M. Guidry, Int. J. Theor. Phys. C9, 745 (1998).

[15] See for example, C. Brennen, Cavitation and Bubble Dynamics (Oxford University Press, New York, 1995).

[16] T. Kodama, H.-Th. Elze, I. Scott and J. Rafelski, Heavy Ion Physics, 5, 390 (1997).

[17] J. von Neumann and R. D. Richtmyer, J. Appl. Phys., 21 (1950), 232.

[18] A.H. Taub, Phys. Rev. 94 (1954), 1468.

[19] B. F. Schutz Jr., Phys. Rev. D2 (1970), 2762.

[20] J. R. Ray, J. Math. Phys. 13 (1972), 1451.

[21] G. Yu. Bogoslovskii, Sov. Phys. Dokl. 31(11) (1986), 879.

[22] C. W. Misner and D. H. Sharp, Phys. Rev.136 (1964), 571.

[23] L. Mittag, M. J. Sephen and W. Yourgrau, in Variational Principles in Dynamics and Quantum Theory, ed. W. W. Yourgrau and S. Mandelstam (Dover, New York, 1968).

[24] J.W. Herivel, Proc. Camb. Phil. Soc. 51 (1955), 344.

[25] C.C. Lin, Proceedings of the International School of Physics, Varenna (New York, 1963).

[26] T. Chiueh, Phys. Rev. E 49 (1994), 1269.

[27] S. Weinberg, Gravitation and Cosmology (John Wiley \& Sons, New York, 1972).

[28] D. Kramer, H. Stephani, E. Herlt and McCallum, Exact Solutions of Einstein's Field Equations, Cambridge University Press (1980). 


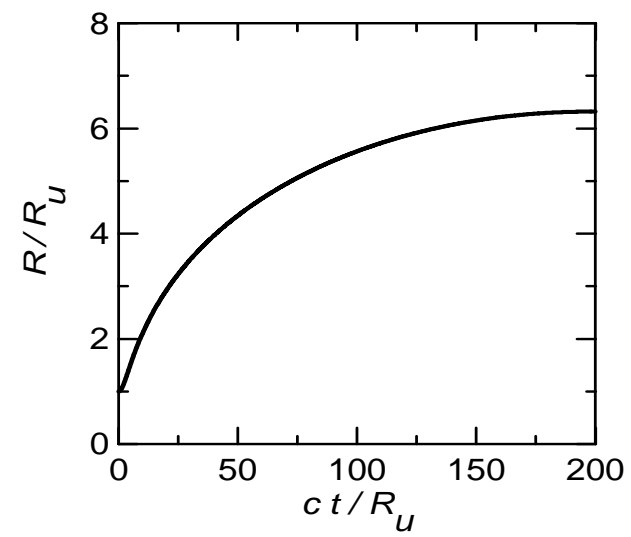

Fig.1-a

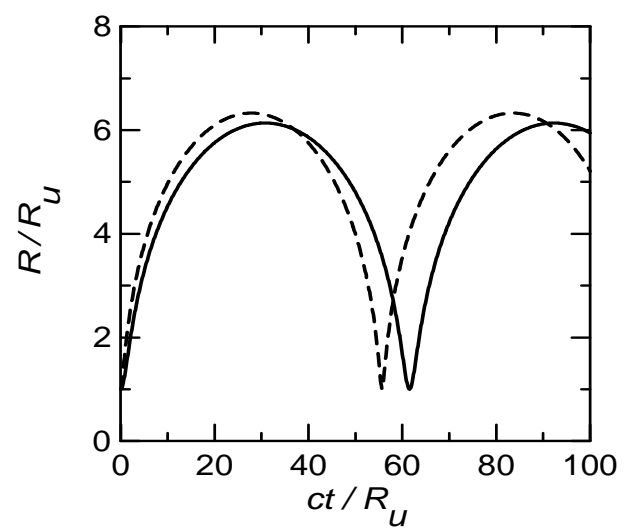

Fig. 2-a

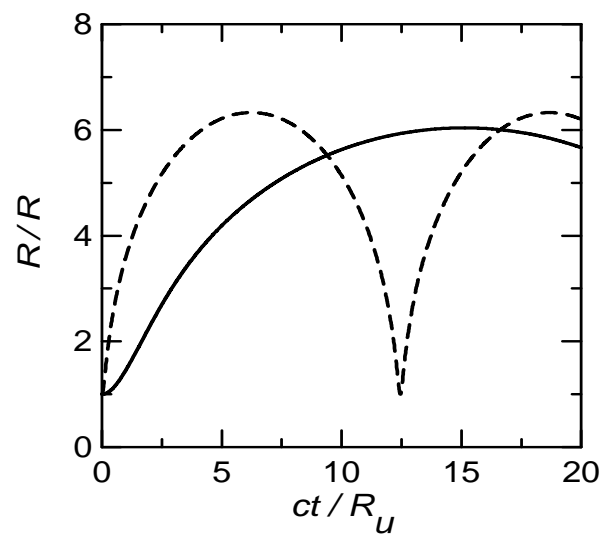

Fig. 3-a

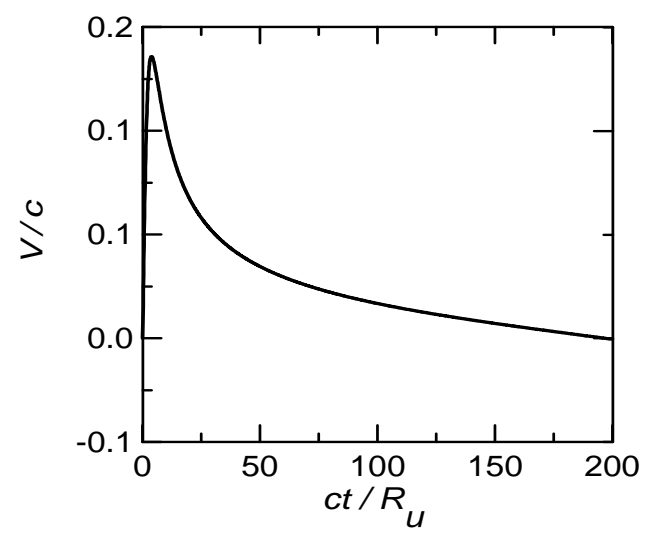

Fig. 1-b

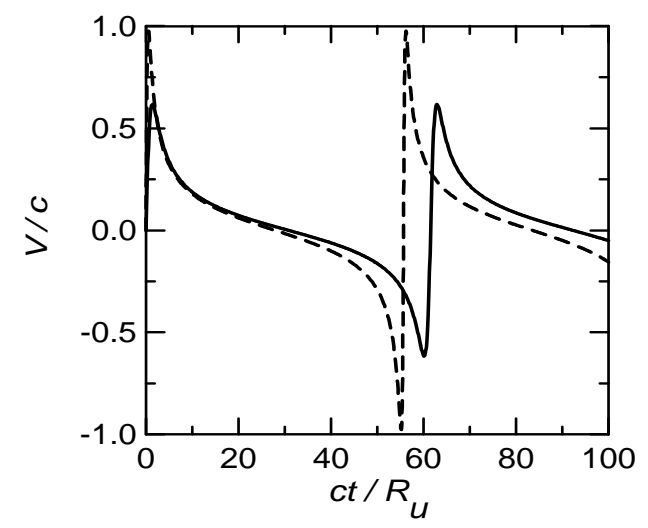

Fig. 2-b

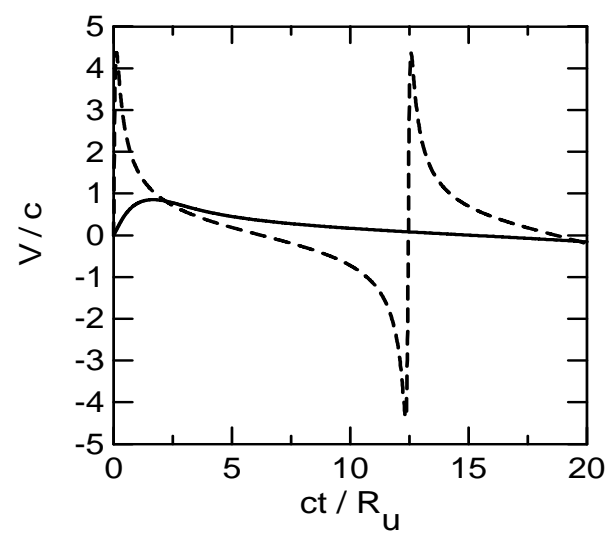

Fig. 3-b 